\title{
Analysis of a Complex Architectural Style C2 Using Modeling Language Alloy
}

\author{
Ashish Kumar Dwivedi*, Santanu Kumar Rath \\ Department of Computer Science and Engineering, National Institute of Technology, Rourkela, 769008, Odisha, India
}

*Corresponding Author: ashish.nitcs@gmail.com

Copyright (C2014 Horizon Research Publishing All rights reserved.

\begin{abstract}
Software architecture plays an important role in the high level design of a system in terms of components, connectors, and configuration. The main building block of software architecture is an architectural style that provides domain specific design semantics. Although many architectural description languages (ADLs) are available in literature for modeling notations to support architecture based development, these ADLs lack proper tool support in terms of formal modeling and visualization. Hence formal methods are used for modeling and verification of architectural styles. In this study, an attempt has been made to formalize one complex style i.e., C2 ( component and connector) using formal specification language Alloy. For consistency checking of modeling notations, the model checker Alloy Analyzer is used. It automatically checks properties such as compatibility between components and connectors, satisfiability of predicates over the architectural structure, and consistency of a style. For modeling and verification of C2 architectural style, one case study on cruise control system has been considered. At the end of this study performance evaluation among different SAT solvers associated with Alloy Analyzer has been performed in order to assess the quality.
\end{abstract}

Keywords Software Architecture, Architectural style, C2 style, Alloy, Alloy Analyzer, Cruise Control System

\section{Introduction}

A software architecture comprises a set of principal design decisions that deals with high-level structure of a system [1]. In the architectural development process, design decisions are usually being represented in terms of structure, behavior, interaction, and non-functional properties of the system. In the present day of software development, the complexity of desired outcomes are increases day by day. The present day, software development activities are shifting the emphasis from lines of code to architectural elements i.e., software components and connectors. Software architect intends to build systems across multiple application domains. He feels that under given conditions, some design decisions occur in solution. An architectural style is an architectural design decisions to capture knowledge of effective designs for achieving specified goals in a given development context [2]. Styles provide a common semantics for a software architect in order to make the design more easily understandable. Many styles are available, such as client-server, virtual machine, pipe-and-filter, blackboard, rule-based, publish-subscriber, event-based, peer-to-peer etc. for the development of a system. As the complexity of the system increases, large number of complex styles have been introduced such as C2 (components and connectors), CORBA, REST architecture etc.

Modeling and development of a software architecture are generally done by architectural description languages (ADLs). There are many ADLs such as, Aesop, C2SADEL, ArTek, Darwin, Rapide, SADL, UniCon, Weaves, Wright etc. available for a software ar- 
chitecture [3]. These ADLs support mathematical notations and tools for modeling different architectural styles and architectural patterns. The use of ADLs vary from one problem to another e.g., Rapide [4] is used to model component interface and external behavior of a system, whereas Wright [5] models an architectural element connector. These ADLs have limitations for tool support in terms of modeling, platform support and formal verification. A number of complex styles have also been introduced for modeling and visualization of complex and heterogeneous systems. The ADLs are not sufficient for modeling and analyzing of complex styles. Because, the complex styles provide a semi-formal notation for the analysis of a complex system. Hence formal methods are being considered for modeling, refinement, and formal verification of software architecture. Formal methods and model checking tools are helpful to support architecture based development.

Formal methods are collection of tools and techniques that can capture the abstract features of a system [6]. Formal specification is used to describe the software requirements precisely and unambiguously. In order to check whether the modeled system complies with the user requirements, it needs to verify and validate the model. A number of analysis techniques such as, reachability analysis, static code analysis, automated theorem proving, model checking etc. are available for testing and evaluating non-functional properties based on the architectural styles. Among them, model checking is a verification technique, used to verify whether an architectural model conforms to expected requirements. Model checking of any software system is the algorithmic analysis of programs to prove properties of their executions. By model checking, important system properties like functional behavior, consistency of internal structure, and performance characteristics are verified. Logic and theorem proving provide the conceptual framework in which the goal is to formalize the fundamental requirements and specify algorithmic procedures for the analysis of requirements [7].

The goal of this study is to analyze C2 style [1] using formal modeling language Alloy [8]. A safety critical system i.e., cruise control system (CCS) [9] has been considered as a case study for designing the architecture in $\mathrm{C} 2$ style. Subsequently Alloy notations of C2 style are analyzed using the model generator Alloy Analyzer [10]. A number of formal models have been proposed for simple styles such as, client-server, publish-subscriber, pipe and filter, event-based etc. It is observed that more rigorous study needs to be carried out for formalization of complex styles such as, C2. ACME [11] is an architectural interchange language used to model a system using different simple architectural styles such as call-return, data- flow, event-based, and repository. It supports mapping of architectural specifications from one ADL to another; but ACME can not modeled system in C2 style. Generally C2SADEL (Software Architecture Description and Evolution Language for C2-style) is used to modeled C2style. The tool known as DRADEL (Development of Robust Architectures using a Description and Evolution Language) [12] supported by C2SADEL provides textual and graphical modeling as well as skeleton generation; but this tool is not sufficient for simulation and formal verification. Presently systems are running in a distributed, heterogeneous environment and software components of a system are written in different languages. Hence, the software components should follow the principle of substrate independence. The C2-style provides a large number of benefits such as, substrate independence, accommodating heterogeneity, support for product lines [13], ability to design in model-view-controller pattern, support for distributed applications [14, 15].

\section{Preliminaries}

\subsection{Formal modeling language Alloy}

Alloy is a formal modeling language for representing structural properties of a system. It provides a state based formula where syntax is powerful enough to express complex constraints. Alloy is used to support a fully automatic semantic analysis that can provide checking of consequences, consistency, and simulated execution [8]. The semantics of Alloy bridges the gap between Z [16] and object models. Alloy is mainly designed to search for instances within finite scope. It offers declaration syntax compatible with graphical object oriented models. Alloy specification is built from atoms and relations. The main building blocks of Alloy modeling language are: Signature, Field, Predicate, Function, Fact, Assertion, Command and Scope. Signature is a collection of fields. The field represents a relation between atoms.

Alloy specification is executed by an automated tool i.e., Alloy analyzer [10] which analyzes the Alloy model. During the execution process, Alloy notations are translated into satisfiability problem using SAT solver. After that SAT solvers are invoked to exhaustively search for satisfying models or counterexamples. In Alloy model, additional constraints can be added as facts and assertions. If any fact does not hold, Alloy Analyzer generates an error message. If an assertion does not satisfy the Alloy Analyzer, produces a counterexample. In order to generate and visualize instances for an Alloy model, a predicate is used. A predicate is a logical formula used for an operation of a system. 


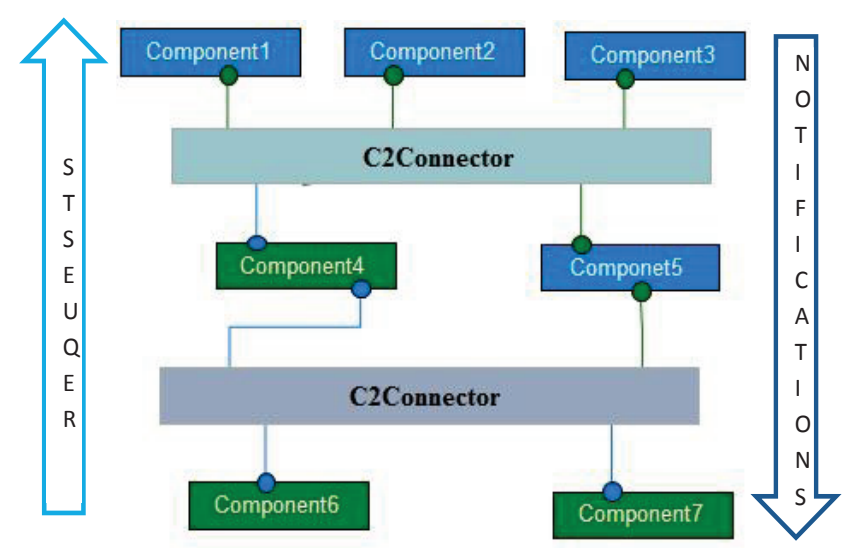

Figure 1. An example of C2 Style

\section{$2.2 \quad$ C2 an architectural style}

C2 is a message-based architectural style for developing flexible and extensible software system. It is based on layered of concurrent components linked by connectors in accordance with a set of rules [17]. Communication among components is done by implicit invocation. The principle of this style is to provide limited visibility among components. A component in a $\mathrm{C} 2$ style is only aware of services provided by components above it in the hierarchy. A component is completely unaware of services provided by components beneath it.

In a $\mathrm{C} 2$ style, a component placed at the bottom layer utilizes the services of components above it by sending a request message. Components on the upper layer emit the notification messages when they change their states. $\mathrm{C} 2$ connectors broadcast notification messages to every component and connector present at the bottom layer. Thus, notification messages represented as an implicit invocation mechanism, enable several components to react to a single component's state change [18]. Figure 1 shows the example of $\mathrm{C} 2$ style developed in a tool known as AcmeStudio. An architectural interchange language models an architectural styles using AcmeStudio. This tool does not support $\mathrm{C} 2$ style. The Figure 1 is designed using an event-based style; because C2 style is much similar to event-based style. Above figure has seven components, two $\mathrm{C} 2$ connectors, and nine links. Component6 and component7 send only request messages to upper layer components, whereas component1, component2, and component 3 broadcast only notification messages to the lower layer components. Component4 and component5 send request messages and broadcast notifications to upper layer components and lower layer components respectively.

\subsection{Component}

A software component is an architectural element that encapsulates processing and data in a system's architecture. It restricts access to a subset of the system's functionality and/or data via an explicitly defined interfaces. It can be deployed independently [19]. A software component has a set of runtime interfaces, known as a port. The port allows the points of interactions between the component and connector. The Alloy notation of the component is given below:

\section{sig Component $\{\quad$ ports : set Port $\}$}

\subsection{Connector}

In a complex and distributed heterogeneous environment interaction may become more important and challenging than the functionality of the individual components. A software connector has the task of effecting and regulating interactions among components. It also provides application-independent interaction facilities. A connector has set of roles that identify the components and connectors in the interaction. The Alloy notation of connector is given below:

$$
\operatorname{sig} \text { Connector }\{\quad \text { roles : set Role }\}
$$

\subsection{Port}

It is not possible in current component models to deal separately with an element of an interaction point when such an element is needed alone for specifying a specific logic [20]. A port defines the points of interaction of a component with its environment. Components with complex interfaces are overloaded with many different ports. The Alloy notation of port is given below:

sig Port $\{\quad$ component : one Component $\}$

\subsection{Role}

In software architecture, components cannot directly connect to connectors. They require a suitable role in connector that are compatible with a port in component. A role helps to facilitate the interaction between a connector and a component. A connector is composed 
of roles that are connected to specific ports. The roles are used to specify which interfaces of the port are being used. The Alloy notation of role is given below:

\section{sig Role $\{\quad$ Connector : one Connector $\}$}

\section{Related Work}

Kim and Garlan [21] have mentioned about mapping of an architectural style into a relational model. They expressed an architectural style using formal modeling language Alloy can be checked for properties such as whether a style is consistent, whether a style satisfies some logical constraints over the architectural structure, whether two styles are compatible for composition, and whether one style refines another or not. Wong et al. [22] presented a technique to support the design and verification of software architectural models using the model checker Alloy Analyzer. They presented use of the architecture style library in modeling and verifying a complex system that utilizes multi-style structures. Heyman et al. [23] illustrated the need of formal modeling techniques for software architect who need to precisely ascertain the security properties of their design models. They have proposed a technique that motivates an architect to easily develop, secured architecture designs by assembling already verified security pattern models.

Keznikl et al. [24] presented an approach for automated resolution of connector architectures based on constraint solving techniques (ARCAS). They used a formal modeling language Alloy for describing a connector theory. They employed a constraint solver to find a suitable connector architecture as a model of the theory. Bertolino et al. [25] illustrated software architecturebased analysis, evaluation, and testing. Zhang et al. [26] described the formal syntax of the Wright architectural description language together with its operational semantics in Labeled Transition System (LTS). They presented an architectural style library that embodies commonly used architecture patterns to facilitate the modeling process. They had considered the Teleservices and Remote Medical Care System (TRMCS), as case study.

Pahl et al. [2] presented an ontological approach for architectural style modeling based on description logic as an abstract, meta-level modeling instrument. They introduced ontologies as a mechanism described and formally defined architectural styles. Hansen and Ingstrup [27] have presented an application of the Alloy modeling language to model architectural change. They demonstrated that it is possible to model architectural change in a relational, first-order language with both a static and dynamic model of architectural runtime structure and architectural runtime change respectively. Bagheri et al. [28] described the feasibility of automated computation of architectural descriptions with an executable prototype developed in Alloy. First, they identified the behavior of architecture as an independent variable. Subsequently a conceptual architecture to make this idea precise, including a graphical notation showing how the key concepts relate to each other.

\section{Proposed Work}

\subsection{Application of C2 style on a case study}

A cruise control system (CCS) has been considered as a case study. It is a safety critical real-time system typically aims to increase passenger safety. It is designed for a car with an automatic transmission. An architectural style $\mathrm{C} 2$ is used in structuring embedded control applications. Figure 2 shows the architecture of cruise control system in C2-style. The components in this style are organized in a layered structure. In this example there are five sensors at the top layer of the cruise control system. The Global positioning system (GPS) senses the location and time information. The axle sensor senses number of pulses per rotation of the axle. The engine sensor senses signals when the engine switches on and off. The brake sensor senses signals when the brake is pressed and another signal when it is released. The wheel revolution sensor senses the number of revolution of wheel.

The component clock generates a pulse when sensors change their state. There is a facility of implicit feedback in such applications via the external environment.

At the next top layer there are four components such as GPS artist, axle artist, engine artist, and brake artist for receiving notifications broadcast from sensors. These components are used to handle information broadcast from different sensors present at the top layer. The artist components receive notifications of different sensors changes, causing them to update their depictions. Artist components maintain the state of a set of abstract graphical objects that, when modified, send state change notifications in the hope that a lower level graphics component will render them on GUI. The cruise controller is the main component that takes data from upper layer components to perform computations. The function of this component is to maintain the speed of a car without interference of user. There is a connection between sensor and controller for receiving notification directly from wheel revolution sensor to cruise con- 
troller to calculate speed and compared it with desired speed. Cruise controller requests for data from sensors to perform computations. By notification messages, sensors broadcast data to it. After performing computation cruise controller broadcast calculated values to different actuators i.e., throttle actuator and GUI. The throttle actuator sends request message for required data and listen to the cruise controller for notifications. In $\mathrm{C} 2$ style, components are independent, concurrent message generators and/or consumers. Whereas connectors are message routers that may filter, translate, and broadcast messages such as requests and notifications.

\subsection{Specifying C2 style of Cruise Control Sys- tem using Alloy}

Modeling a software or a hardware system using formal method Alloy has several advantages. Alloy provides formal model in an executable form which ensures that model has unambiguous and testable semantics. Model generator Alloy Analyzer translates high-level, declarative, relational expressions of the formal model into a SAT formula that can be solved by different SAT solvers such as, SAT4J, Zchaff, MiniSAT etc. [29]. Alloy is also helpful to visualize a model of unbounded size and later specifies a size in a bounded form when verifying properties. To make the explanation more precise, formal method Alloy is used for specifying essential properties of the cruise control system represented in $\mathrm{C} 2$ style. Behavioral properties of cruise control system can be expressed as a form of logical predicates which can be analyzed by using the tool Alloy Analyzer [30].

The following Alloy specification of cruise control system has a module Cruise_Control_System to split a model among several modules. A module in Alloy allows constraints to be reused in different contexts. There are four enumerations such as, FuelLevel, Speed, Brake, and Accelerator have been considered. Like a signature, enumeration can also contain a set of atoms. In the process of analysis, Alloy Analyzer selects all instances for the given scope. Therefore the number of atoms become very large that an explicit enumeration would be infeasible. Alloy Analyzer uses pruning techniques (analysis technique) in order to rule out whole sets of atoms at once. First enumeration FuelLevel is used to specify fuel level of engine. Enumerations Speed, Brake, and Accelerator are used to indicate the status of speed (LowSpeed or ConstSpeed or HighSpeed), state of brake (ON or OFF), and state of accelerator (Pushed or UnPushed) respectively.

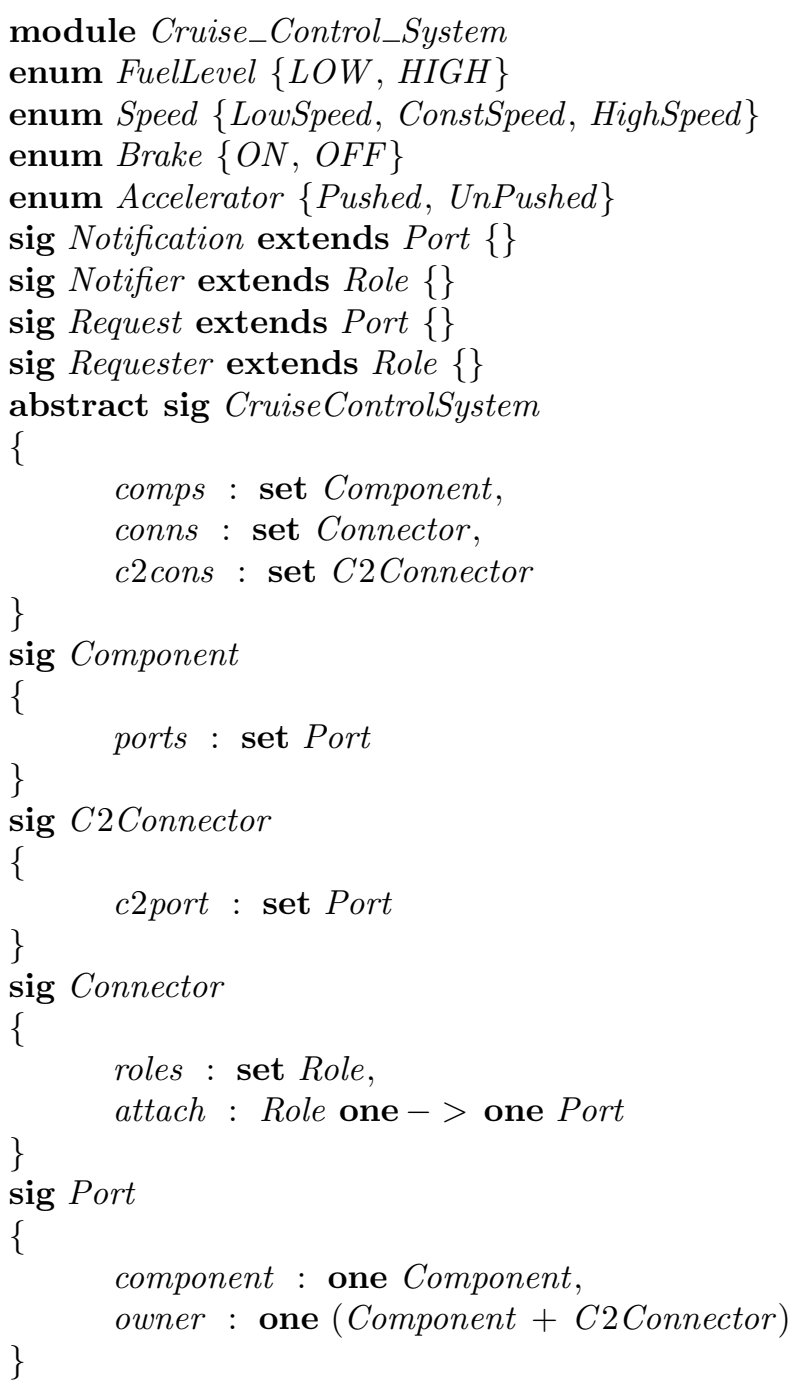




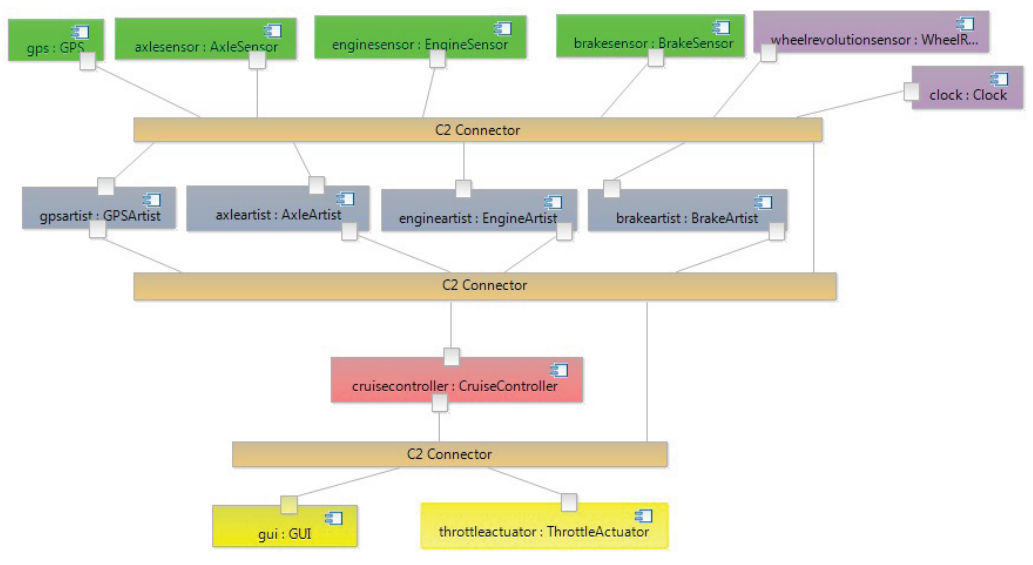

Figure 2. Cruise Control System in C2 architectural style

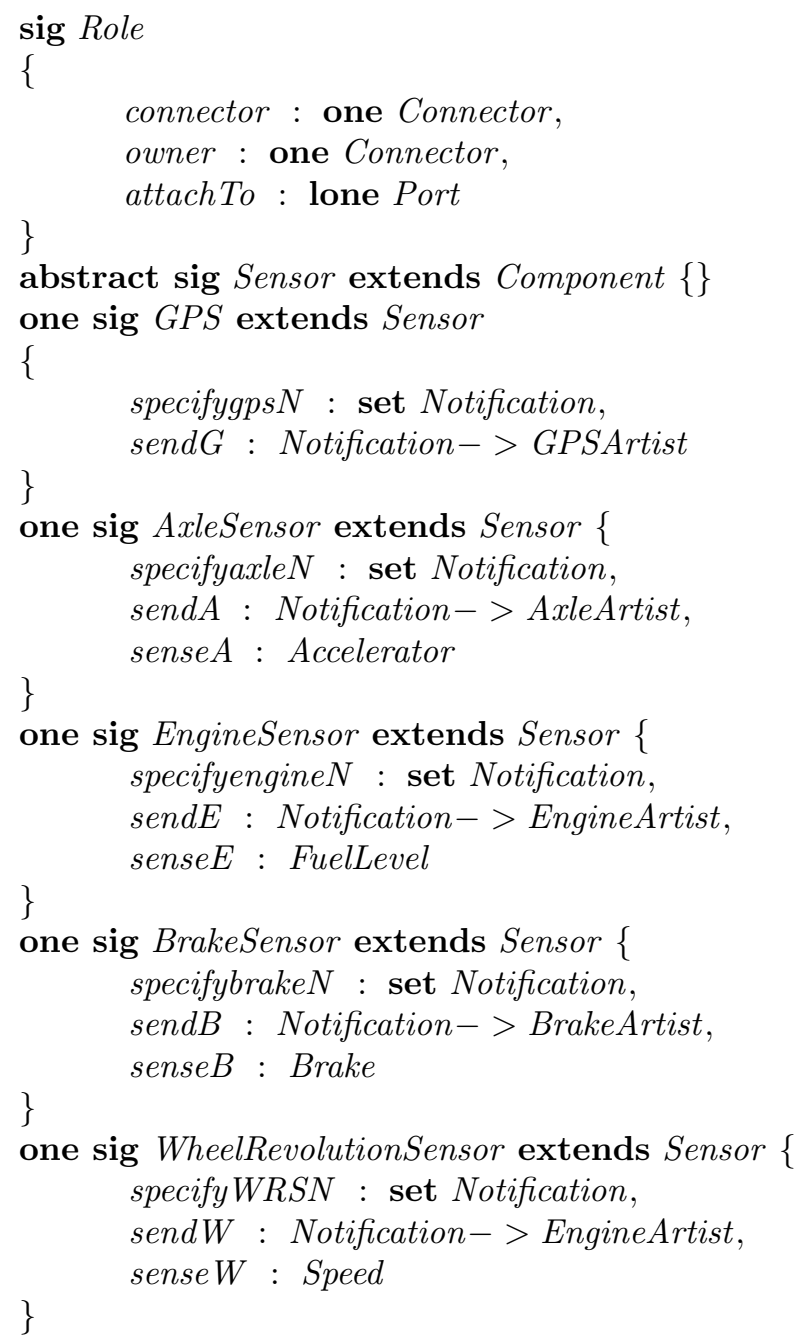

In this Alloy specification, four signatures have been considered such as, Notification, Notifier, Request, and Requester for communication among the components using message passing. Message passing between components is only done by request and notification messages. In this Alloy model, another signature is a CruiseControlSystem which represents the whole system in terms of components, connectors, and C2-connectors. An architectural style is a collection of components and connectors. Each component has a set of ports to connect with different connectors. Similarly, each connector has a set of roles to connect with the ports of a component. The field owner in the port and role signatures indicates that each role and each port have single owner; because a port is owned by single component and a role is owned by single connector.

The C2-style architecture of cruise control system has five sensor components such as, GPS, BrakeSensor, AxleSensor, EngineSensor, and WheelRevolutionSensor. These components are placed on the top layer in the hierarchy. Hence, these sensor components broadcast notification messages and receive request messages. GPS component has two fields such as, specifygps $N$ and sendG. Field specifygps $N$ indicates that a GPS component specifies a number of notifications for other components which are placed into bottom layers and field send $G$ indicates that notifications are received by GPSArtist component. AxleSensor component has three fields such as, specifyengineN, sendE, and senseE. First two fields work same as in GPS component, whereas third field senseE indicates the fuel level. BrakeSensor component also has three fields such as specifybrakeN, sendB, and senseB. The third field, sense $B$ is used to indicate the status of brake. WheelRevolutionSensor component also contains three fields such as, specify$W R S N$, send $W$, and sense $W$. The field sense $W$ indicates 
the speed of a vehicle.

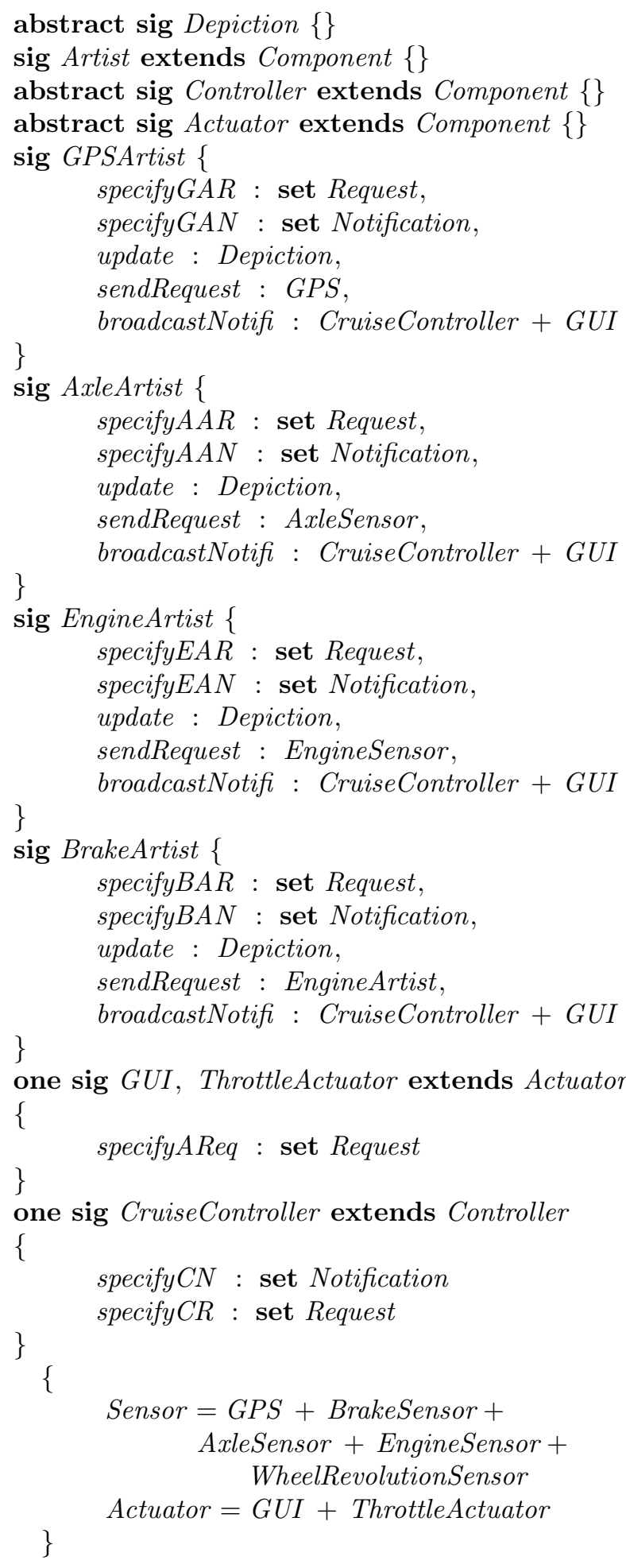

Four artist components are specified in the above Alloy specification such as, GPSArtis, AxleArtist, En-
gineArtist, and BrakeArtist for maintaining the states of abstract graphical objects. The artist components receive notification messages of sensor's state change, causing them to update their depiction. GPSArtist component has five fields such as specify $G A R$, specify $G A N$, update, sendRequest, broadcastNotifi. The first field specify $G A R$ represents a set of request messages for the top layer components. The field specify $G A N$ represents a set of notification messages for bottom layer components. The Third field update indicates the state changed of sensor component in the form of depiction. In architectural style $\mathrm{C} 2$, a component has all requisite information about the upper layer components, whereas it has no information about bottom layer components. Hence, an artist components send request messages to a specific upper layer component and broadcast notifications to all components placed at the layer below it, from that component's layer. Fourth field sendRequests indicates request messages sent from this artist component to only GPS component. Whereas broadcastNotifi field represents notification message sent from this component to controller and actuator components. Similarly, other artist components also have five fields for showing relationship with other components.

In C2-style of cruise control system, cruise controller is the main component. Hence, cruise controller sends and broadcasts both requests and notifications to the upper layer components as well as lower layer components respectively. It has two fields such as specify $C N$ and specify $C R$ in order to represent a set of notifications and requests respectively. C2-style of cruise control system has two actuators such as, GUI and throttle actuator for receiving data, sent from upper layer components. These actuators can specify only requests messages, because the bottom layer components send only request messages to upper layer components. In formal method Alloy, ' +' operator is used for the union operation. Hence, sensor shows the union of all sensor components and actuator shows the union of all actuator components those are used in this style.

\subsection{Analysis of dynamic behavior of architec- tural style C2}

In the process of model checking, analysis is a form of constraint solving. Analysis can disclose subtle flaws that software architect might not have discovered until much later. In Alloy, constraints can be used with the help of fact. A fact is a logical constraint that should always hold. By adding fact statements, checking assertions and executing a predicate the analysis problem can be reduced. In this Alloy model, many facts have been specified. In Alloy model of cruise control system, 
portroleowner is a first fact. It indicates that if a port is present in the component, it means that this port is owned by the component and the component is the owner of this port. Similarly, if a role is present in the connector, it means that this role is owned by the connector and the connector is the owner of this role. In the first fact, name is given but in second fact name is not specified. In Alloy, fact name is optional. The second fact indicates that if some roles are related to some ports then these roles should be specified by some connectors.

According to the principles of C2-style, two components cannot directly be connected. If one component wants to communicate with other components, it must be connected through a $\mathrm{C} 2$-connector. In $\mathrm{C} 2$-style architecture, C2-connector is a combination of more than one simple connector. Hence, it can be viewed that $\mathrm{C} 2-$ connector acts as a component having a set of ports to connect with other simple connectors having set of roles. Assertion No_comp_connect_comp, analyzes that, if some roles are attached to other some ports and the first port owned by any component and also the second port is owned by any other component, Alloy Analyzer generates counterexamples.

There are certain constraints that a developer does not want to record them as facts. If a developer wants to analyze the model with other constraints, and also to check whether these constraints are related to some other constraints or not. Predicate expressions are used to achieve all these. A predicate is a logical formula with declaration parameters. Predicate describes a set of states and transitions, by using constraints among signatures and their fields. Without using predicate, instances cannot be generated for operation except from counterexample. Following code have two predicates such as Port_Connect_Role and Comp_Connect_C2Con to specify port-role connection and component to C2-Connector attachment operations. Predicate Port_Connect_Role is used for a port and role, returning value true if they are directly connected. In predicate Comp_Connect_C2Con, constraints are added to connect component and $\mathrm{C} 2$-Connector. The keyword disj is used to restrict the bindings and include ones in which the bound variables are disjoint from one another. In this specification, disj indicates that between two roles only one is used. In the second predicate, first predicate is used, because predicates in Alloy act as built-in functions and it can be easily used by other predicates.

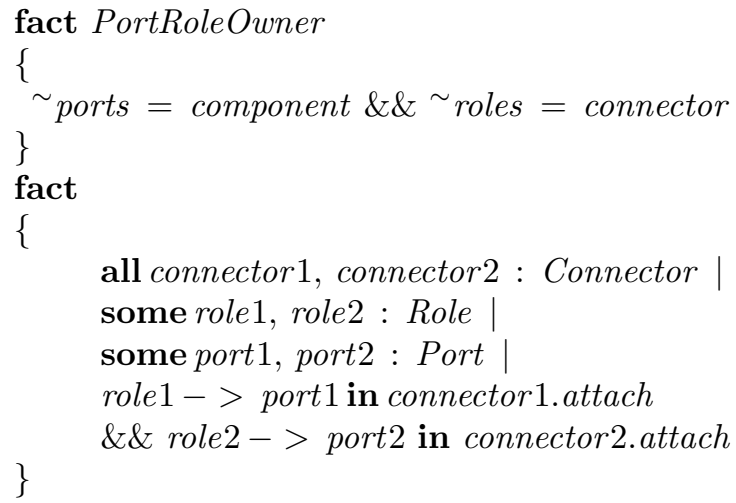

pred Port_Connect_Role [role : Role, port : Port] \{

role $->$ port in Connector.attach

\}

pred Comp_Connect_C2Con [comp : Component, c2con : C2Connector]

\{

some role 1 , role 2 : Role |

some port 1 , port 2 : Port |

$\operatorname{disj}[$ role 1 , role 2$] \& \&$

Port_Connect_Role[role1, port 1$]$

$\& \&$ owner $[$ port 1$]=$ comp

\&\& Port_Connect_Role[role2, port2]

$\& \&$ owner $[$ port 2$]=c 2$ con

$\& \&$ owner $[$ role 1$]=$ owner $[$ role 2$]$

\}

For cruise control system some constraints are usually added in the form of facts. The fact axle_sensor_Notification ensures that if speed of vehicle is high and accelerator is not pushed then axle sensor component sends notification message to artist component. Similarly engine_sensor_Notification fact ensures that if the value of speed is low-speed and fuel-level is 
also low, then engine sensor component broadcast notification message to artist component.

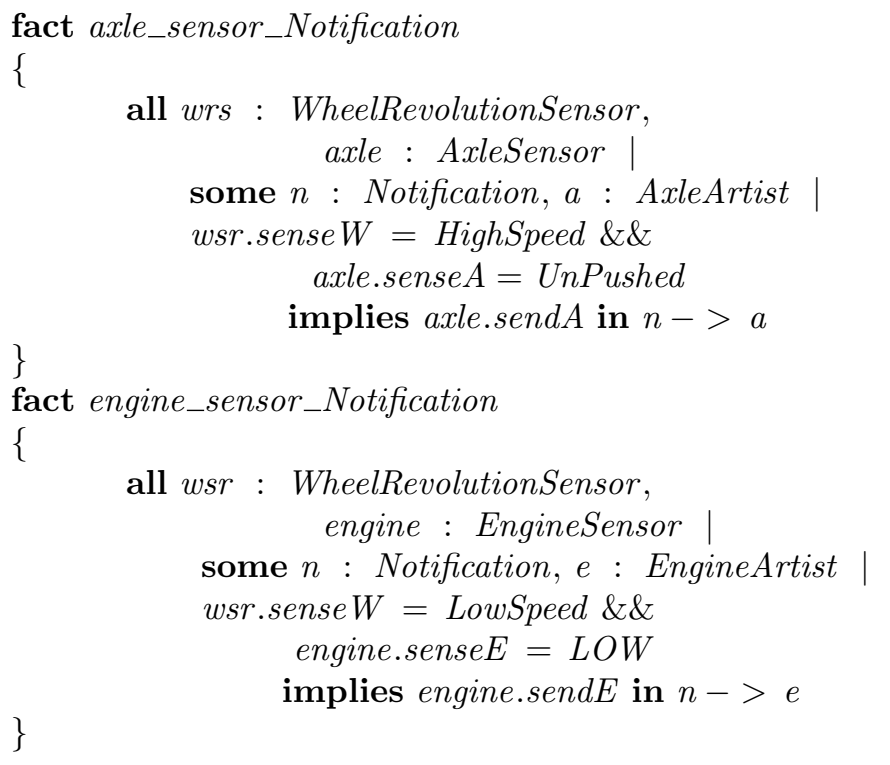

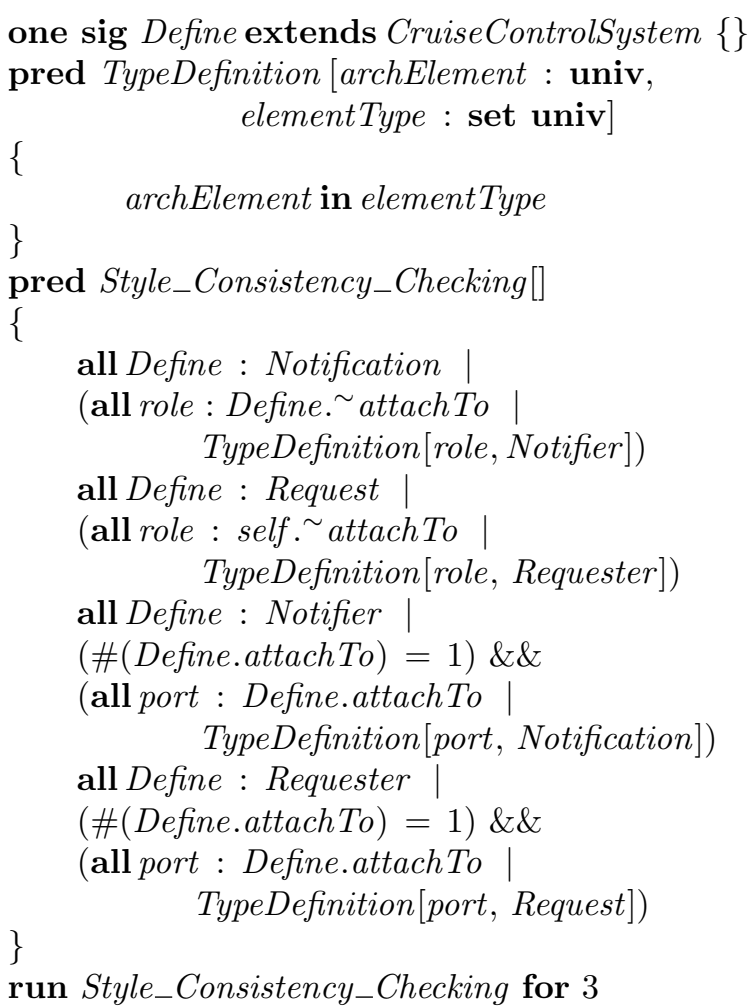

In the following Alloy model, Define signature is used to restrict the model to generate only one system instance. The predicate Type-Definition specifies the definition of architectural elements and their types. Keyword univ is an unary operator represented as universal set. Style_Consistency_Checking predicate specifies the type definition of notification and request signatures. In this study, Notification and Request are considered as a port and Notifier and Requester are considered as a role. First constraint considers Define as a Notification attached by a Notifier (it is a type of role). Similarly, in next the constraint, Define is considered as Request attached by a Requester. Other two constraints are inversely related to first two constraints.

Third constraint considered Define as a role (Notifier) to attach with the port Notification. In this constraint, it is specified that the number of link between a role (Notifier) and a port (Notification) should be one. Fourth constraint is similar to third constraint for port request and role requester.
In order to generate and visualize instances, execution of the run command is done by clicking the execute button. After finishing execution, Alloy analyzer indicates that it has found instances which can be visualized by clicking on the show button. On clicking show button, Alloy Analyzer generates instances according to given scope. Operation Style_Consistency_Checking is visualized for the scope value three, which means that Alloy Analyzer generates at most three instances of each atoms. The pictorial representation of this predicate is shown in Figure 3. This figure shows different objects as enumerations, signatures, and connection between these signatures representing a relation. It is possible to increase the number of instances by using a keyword scope in the run command. If scope is not defined in run command then by default, Alloy Analyzer assumes the value of scope as three. As literature says if the value of scope is more than seven, then Alloy Analyzer generates all possible types of relations among given objects. There is a button next in Alloy Analyzer which shows all possible types of relations among the objects. Figure 4 shows some of the types used in our expression together with the relations between these types. This meta model provides conceptual map of our model. 


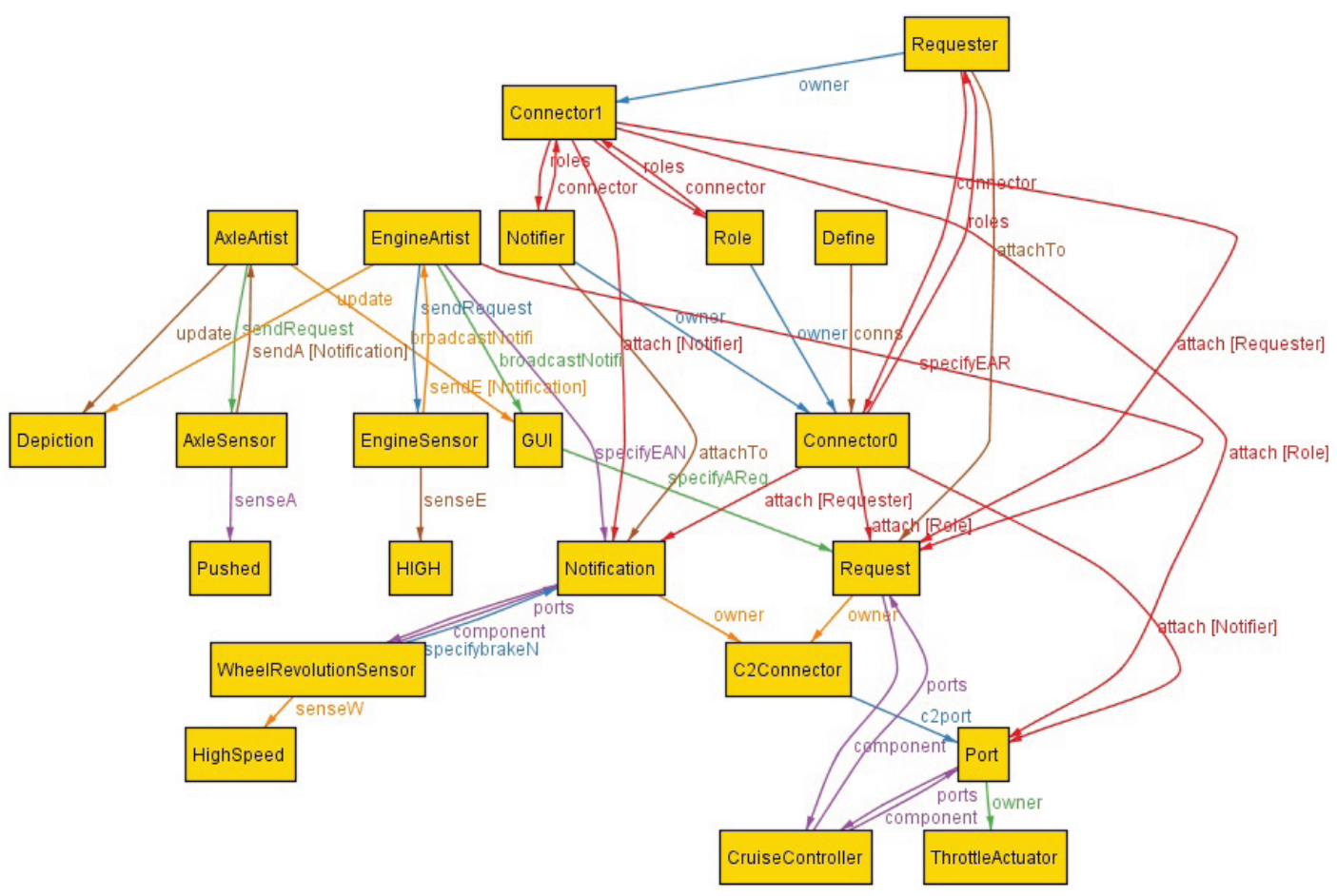

Figure 3. Instances generated by Alloy Analyzer

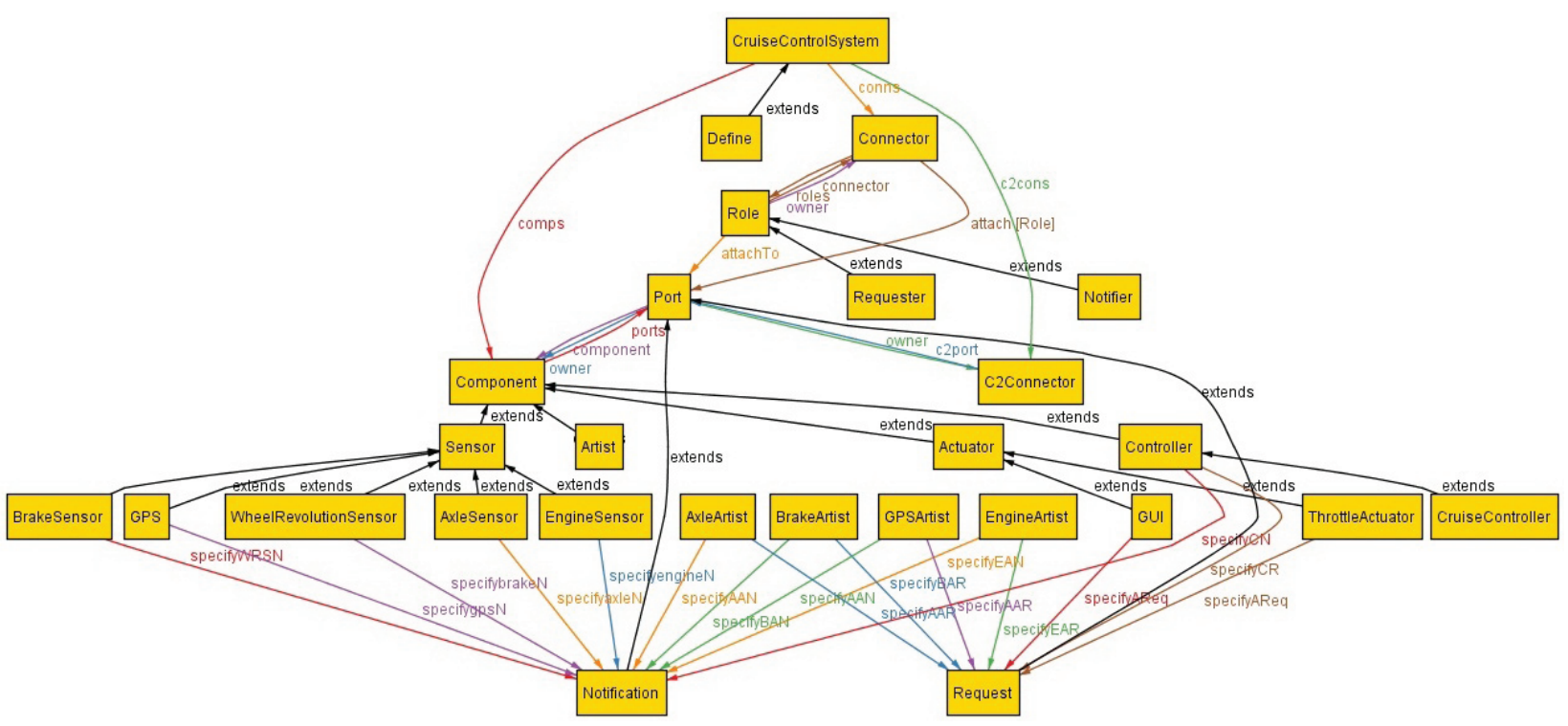

Figure 4. Meta model of Cruise Control System 


\subsection{Performance evaluation for different SAT solvers}

To analyze the performance of analysis, the consistency on Alloy specification of cruise control system considering problem size from 2 to 12 has been performed. For the performance evaluation, system configuration is Intel(R) Core(TM) i5-2400 CPU @ $3.10 \mathrm{GHz}, 2.00$ GB (1.88 GB usable), 32-bit Windows 7 operating system. Execution is carried out using Alloy Analyzer 4.2, build date: 2012-04-20 10:05 EDT. During execution process, SAT solver is SAT4J where maximum stack to use was $8192 \mathrm{k}$ and maximum memory to use was $768 \mathrm{M}$. For problem size 2 to 10 above details are used; when the execution performed for problem size 12, Alloy Analyzer generates an error message related to memory used. Hence for problem size 12 , maximum memory $1024 \mathrm{M}$ is used. The performance result for different bound range (from 2 to 12) is shown in Figure 5. As shown in Figure 5 , for problem size 12 time reach its limit of tractability for C2-style.

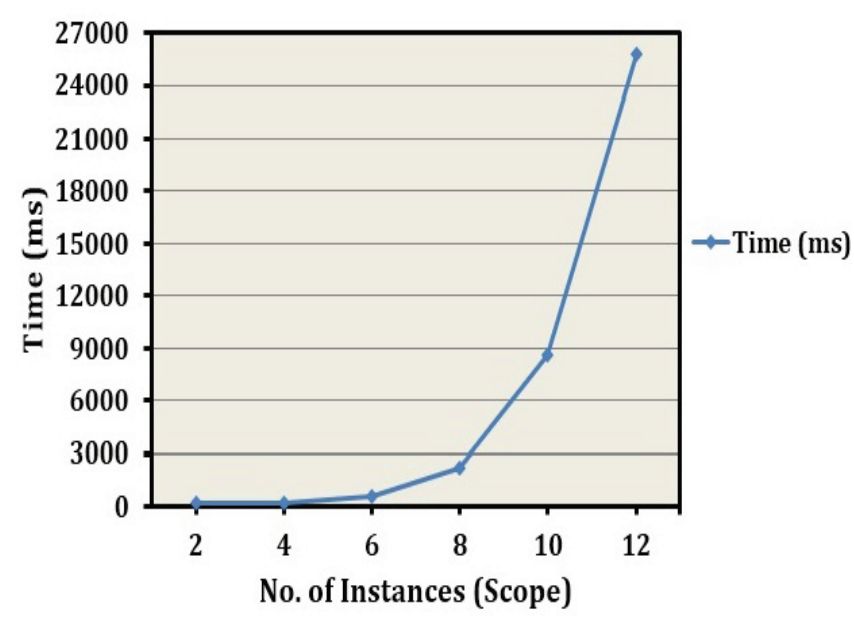

Figure 5. Performance evaluation for SAT4J solver

The Alloy Analyzer supports many SAT solvers such as MiniSat, MiniSat with Unsat Core, ZChaff, and SAT4J to exhaustively search for satisfying models or counterexamples. The comparative analysis among these solvers for bound range 7 , maximum memory used 768 , maximum stack used $8192 \mathrm{k}$ is presented in table:1. Finally the comparison of performance evaluation among these solvers is also presented in Figure 6 for bound range from 2 to 10 with same system configuration.

There are many advantages of analyzing different levels of abstractions of an architectural style such as in-
Table 1. Performance analysis for different SAT solvers

\begin{tabular}{|c|c|c|c|}
\hline S. No. & SAT Solver & Time $(\mathbf{m s})$ & No. of vars. \\
\hline 1. & MiniSat & 982 & 134306 \\
\hline 2. & MinisatProver & 1407 & 134305 \\
\hline 3. & ZChaff & 915 & 134306 \\
\hline 4. & SAT4J & 1104 & 134306 \\
\hline
\end{tabular}

ternal functioning of component and connector, topology of architectural elements, and principle of the architectural style. The first advantage is to provide more understandability for developer to implement different components and connectors. Second advantage is to provide a framework that is helpful for deployment process. Third advantage provides an appropriate level of granularity for accessing non-functional properties of a software system. The use of formal modeling techniques ensures the correctness of any architectural changes performed by an architect. In this study, it is inferred that analysis of dynamic aspects of any style needs to be carried out to assess the correctness.

\section{Conclusion}

In this paper, a study on a safety critical system called as cruise control system using a complex architectural style C2 is presented. Subsequently a library of styles is presented using formal modeling language Alloy to assist the reuse and extensible modeling of complex and highly distributed components, developed in different programming languages. This style library is used to model the cruise control system. Compatibility among components, connectors, and C2-connectors has been checked using formal verifier Alloy Analyzer. Finally, performance evaluation among different SAT solvers have been performed in order to assess the efficiency of Alloy Analyzer. In this study, Alloy is chosen because it provides a compact model that allows the verification of structural and behavioral properties of a system. Modeling the structural properties of an architectural style has generally been associated with the component-connector abstractions. Styles are generally used to promote design reuse, code reuse, and support interoperability between two different styles. Hence, from the above study, it is concluded that, formalizing an architectural style provides style consistency and validity of configuration. It also helps in refinement of critical processes and checking compatibility among different style.

This approach can be extended by considering the application of different models in other complex architectural styles such as, CORBA (Common Object Request Broker Architecture), and REST (REpresen- 


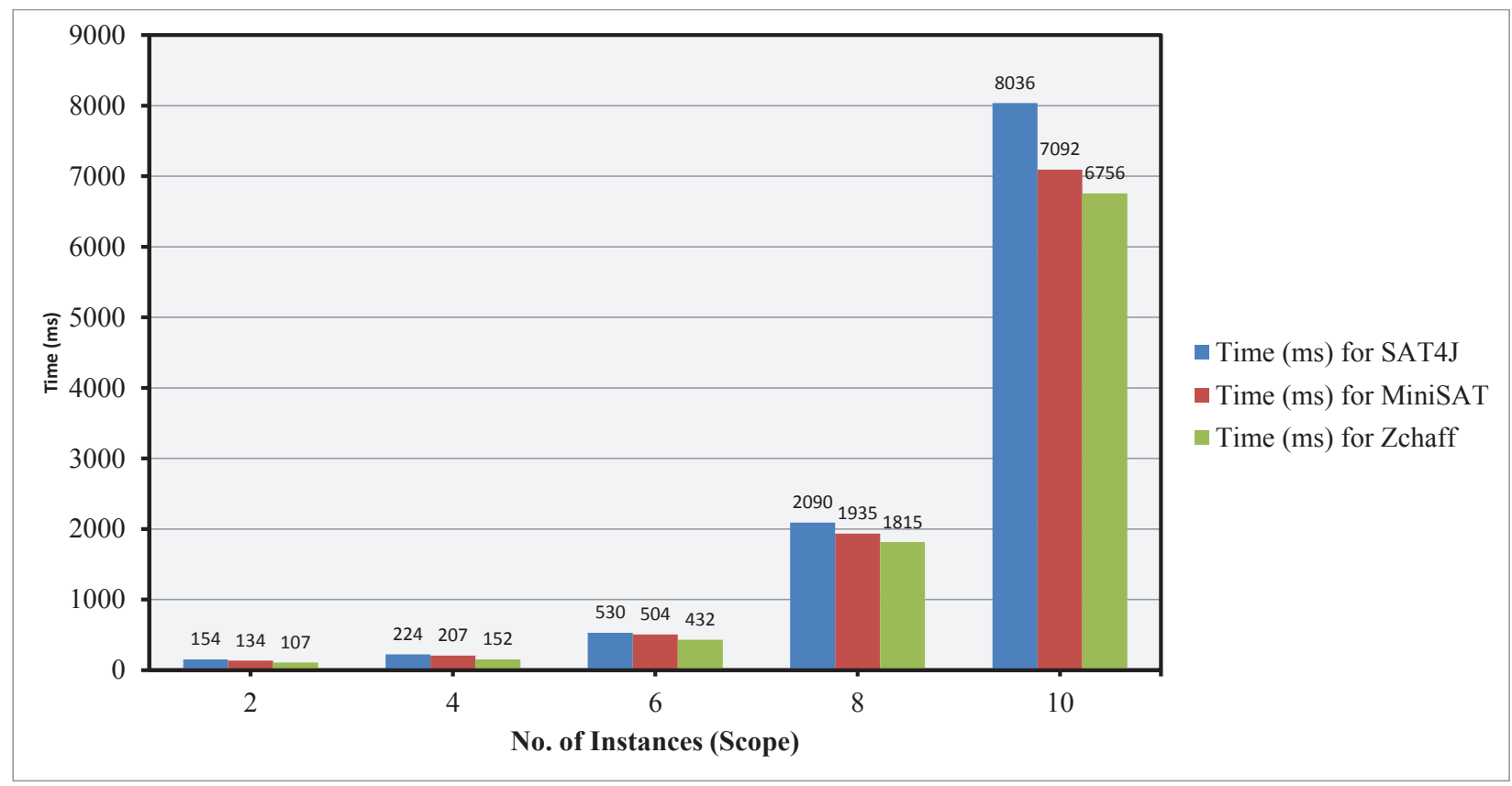

Figure 6. Performance evaluation for different SAT solvers

tational State Transfer) architecture etc. The formal models can be verified using model checkers such as Alloy Analyzer, CPN Tools, and PAT (Process Analysis Toolkit). The verification process may also be carried out considering different architectural patterns such as state-logic-display, Model-View-Controller (well known as MVC pattern), and Sense-Compute-Control etc.

\section{REFERENCES}

[1] R. N. Taylor, N. Medvidovic, and E. M. Dashofy. Software architecture: foundations, theory, and practice. Wiley Publishing, 2009.

[2] C. Pahl, S. Giesecke, and W. Hasselbring. Ontologybased modelling of architectural styles. Information and Software Technology, 51(12):1739-1749, 2009.

[3] N. Medvidovic and R. N. Taylor. A classification and comparison framework for software architecture description languages. IEEE Transactions on Software Engineering, 26(1):70-93, 2000.

[4] D. C. Luckham, J. J. Kenney, L. M. Augustin, J. Vera, D. Bryan, and W. Mann. Specification and analysis of system architecture using rapide. IEEE Transactions on Software Engineering, 21(4):336-354, 1995.
[5] R. Allen and D. Garlan. A formal basis for architectural connection. ACM Transactions on Software Engineering and Methodology (TOSEM), 6(3):213-249, 1997.

[6] J. Woodcock, P. G. Larsen, J. Bicarregui, and J. Fitzgerald. Formal methods: Practice and experience. ACM Computing Surveys (CSUR), 41(4):19, 2009.

[7] R. Jhala and R. Majumdar. Software model checking. ACM Computing Surveys (CSUR), 41(4):21, 2009.

[8] D. Jackson. Software Abstractions: logic, language, and analysis. MIT press, 2006.

[9] H. Gomaa. Software Design Methods for Concurrent and Real-Time Systems. Addison-Wesley, 2001.

[10] S. D. Group. Alloy analyzer 4. http://alloy.mit.edu/ alloy4/, 2010.

[11] D. Garlan, R. Monroe, and D. Wile. Acme: an architecture description interchange language. In Proceedings of the 1997 conference of the Centre for Advanced Studies on Collaborative research, pages 159-173. IBM Press, November 1997.

[12] N. Medvidovic, D. S. Rosenblum, and R. N. Taylor. A language and environment for architecture-based software development and evolution. In Software Engineering, 1999. Proceedings of the 1999 International Conference on, pages 44-53. IEEE, 1999. 
[13] J. Kofroň, F. Plášil, and O. Šerỳ. Modes in component behavior specification via EBP and their application in product lines. Information and Software Technology, 51(1):31-41, 2009.

[14] S. Malek, N. Medvidovic, and M. Mikic-Rakic. An extensible framework for improving a distributed software system's deployment architecture. IEEE Transactions on Software Engineering, 38(1):73-100, 2012.

[15] N. Medvidovic and G. Edwards. Software architecture and mobility: A roadmap. Journal of Systems and Software, 83(6):885-898, 2010.

[16] J. M. Spivey. The $Z$ notation: a reference manual. Prentice Hall International (UK) Ltd., 1992.

[17] H. Muccini, M. Dias, and D. J. Richardson. Software architecture-based regression testing. Journal of Systems and Software, 79(10):1379-1396, 2006.

[18] P. Oreizy, M. M. Gorlick, R. N. Taylor, D. Heimhigner, G. Johnson, N. Medvidovic, A. Quilici, D. S. Rosenblum, and A. L. Wolf. An architecture-based approach to self-adaptive software. Intelligent Systems and Their Applications, IEEE, 14(3):54-62, 1999.

[19] I. Crnkovic, S. Sentilles, A. Vulgarakis, and M. R. Chaudron. A classification framework for software component models. IEEE Transactions on Software Engineering, 37(5):593-615, 2011.

[20] D. Bennouar, T. Khammaci, and A. Henni. A new approach for component's port modeling in software architecture. Journal of Systems and Software, 83(8):1430 1442,2010

[21] J. S. Kim and D. Garlan. Analyzing architectural styles. Journal of Systems and Software, 83(7):1216-1235, July 2010 .

[22] S. Wong, J. Sun, I. Warren, and J. Sun. A scalable approach to multi-style architectural modeling and verification. In Proceedings of the 13th IEEE International Conference on on Engineering of Complex Computer Systems, ICECCS '08, pages 25-34, Washington, DC, USA, 2008. IEEE Computer Society.
[23] T. Heyman, R. Scandariato, and W. Joosen. Reusable formal models for secure software architectures. In Proceedings of the 2012 Joint Working IEEE/IFIP Conference on Software Architecture and European Conference on Software Architecture, WICSA-ECSA '12, pages 4150, Washington, DC, USA, 2012. IEEE Computer Society.

[24] J. Keznikl, T. Bureš, F. Plášil, and P. Hnětynka. Automated resolution of connector architectures using constraint solving (ARCAS method). Software and Systems Modeling, Springer, pages 1-30, 2012.

[25] A. Bertolino, P. Inverardi, and H. Muccini. Software architecture-based analysis and testing: a look into achievements and future challenges. Computing, Springer-Verlag, 95(8):1-16, 2013.

[26] J. Zhang, Y. Liu, J. Sun, J. S. Dong, and J. Sun. Model checking software architecture design. In Proceedings of the 2012 IEEE 14th International Symposium on HighAssurance Systems Engineering, pages 193-200, Washington, DC, USA, 2012. IEEE Computer Society.

[27] K. M. Hansen and M. Ingstrup. Modeling and analyzing architectural change with alloy. In Proceedings of the 2010 ACM Symposium on Applied Computing, SAC 10, pages 2257-2264, New York, NY, USA, 2010. ACM.

[28] H. Bagheri, Y. Song, and K. Sullivan. Architectural style as an independent variable. In Proceedings of the IEEE/ACM international conference on Automated software engineering, ASE 10, pages 159-162, New York, NY, USA, 2010. ACM.

[29] M. W. Moskewicz, C. F. Madigan, Y. Zhao, L. Zhang, and S. Malik. Chaff: Engineering an efficient SAT solver. In Proceedings of the 38th annual Design Automation Conference, New York, NY, USA, pages 530535. ACM, 2001.

[30] D. Jackson. Alloy: a lightweight object modelling notation. ACM Trans. Softw. Eng. Methodol., 11(2):256290, Apr. 2002. 